\title{
Antimicrobial Stewardship with Pharmacist Intervention Improves Timeliness of Antimicrobials Across Thirty-three Hospitals in South Africa
}

\author{
Angeliki P. Messina · Dena van den Bergh · Debra A. Goff
}

To view enhanced content go to www.infectiousdiseases-open.com

Received: June 12, 2015 / Published online: September 11, 2015

(C) The Author(s) 2015. This article is published with open access at Springerlink.com

\begin{abstract}
Introduction: Ensuring timely administration of antimicrobials is critical in the management of patients with infections. Mortality increases by $7.6 \%$ for every hour of delay in the administration of antimicrobial therapy in patients with sepsis. The time elapsed from the written antibiotic order to actual intravenous administration or 'hang-time' can often be several hours due to logistics within the hospital. Our purpose is to evaluate the change in compliance with administering antimicrobials within an hour of prescription after implementation of a national antibiotic stewardship pharmacist-driven hang-time process improvement protocol.
\end{abstract}

Methods: This was a prospective multicenter study in 33 South African hospitals from 1 July 2013-30 August 2014. Two pilot sites established the mechanism for noninfectious

A. P. Messina - D. van den Bergh

Department of Quality Leadership, Netcare

Hospitals Ltd., Johannesburg, South Africa

D. A. Goff $(\bowtie)$

Department of Pharmacy, The Ohio State University

Wexner Medical Center, Columbus, OH, USA

e-mail: Debbie.goff@osumc.edu disease pharmacists to make interventions and document hang-time data. Following this, a hang-time compliance assessment was initiated using the tools of healthcare improvement spread methodology. This consisted of five stages and an implementation toolkit was developed. The pharmacist study coordinator was responsible for implementation, the development of an implementation toolkit and real-time coordination of data with monthly feedback to all sites.

Results: A total of 32,985 patients who received intravenous antibiotics were assessed for hang-time compliance with first doses of new antibiotic orders. Over the 60-week period, 21,069 patients received antibiotics within an hour following prescription and were assessed as hang-time compliant. The change in improvement of hang-time compliance following implementation of a pharmacist-driven hang-time process improvement protocol was $41.2 \%$ pre-intervention week $1(164 / 398)$ to $78.4 \%$ post-intervention week $60(480 / 612 ; P<0.0001)$. Pharmacists reviewed and evaluated twice as many patients during the final 4 weeks (1680) compared to the first 4 weeks (834; $P<0.0001)$. 
Conclusion: Noninfectious disease pharmacists can significantly improve the timely administration of antimicrobials and contribute to low-hanging-fruit antimicrobial stewardship initiatives within a hospital system in a resource-limited country.

Keywords: Antimicrobials; Antimicrobial stewardship; Pharmacist; South Africa

\section{INTRODUCTION}

In the management of patients with severe sepsis and septic shock, every hour of delay in the administration of antimicrobial therapy increases mortality by $7.6 \%$ [1]. Numerous publications support the timely administration of antimicrobials to assist in the improvement of patient outcomes. The majority of these studies are conducted in patients with severe sepsis and septic shock following the onset of hypotension and demonstrate an increase in in-hospital mortality following a delay in antibiotic administration [1-3]. Kumar et al. revealed that this "mortality effect" is not influenced by a particular bacterial pathogen nor hospital versus community epidemiology and is applicable to large patient population groups. Furthermore, a study conducted across 65 intensive care units (ICUs) analyzing 17,990 septic patients revealed that patient outcomes were negatively impacted regardless of illness severity or hospital location when antimicrobial therapy was delayed [2].

The time elapsed from the written antibiotic order on a paper chart to actual intravenous (IV) administration or 'hang-time' can often be several hours because of work flow logistics within the hospital, the pharmacy department and nursing. The following barriers are common reasons for the delay of antibiotic administration within the recommended $1 \mathrm{~h}$ window of time in South African hospital settings: use of paper charts, delays in delivery of the prescription order to the pharmacy, delays in prescription chart assessment, processing time in pharmacy, delivery delays in the transportation of medication from the pharmacy to the wards and reconstitution of IV antibiotics by nursing staff at the patient bedside.

Ensuring the first dose antimicrobial is available at the time of required administration, without delay, is not only a good practice supported by the literature but also forms the foundation of any antimicrobial stewardship (AMS) program as the practice of AMS ultimately aims to optimize antimicrobial therapy to improve patient outcomes [4]. Hang-time, referred to as the "golden hour" of antibiotic administration, is a fundamental process that relies extensively on the involvement, collaboration and participation of all healthcare team members including doctors, pharmacists, nursing staff and hospital management. As a result, it provides a multidisciplinary platform to augment the awareness required when initiating and driving an antibiotic stewardship program in a hospital.

The objective of this study is to measure the change in compliance with i.v. antimicrobial administration within $1 \mathrm{~h}$ following implementation of a national antibiotic stewardship pharmacist-driven hang-time process improvement protocol across a network of South African hospitals.

\section{METHODS}

This was a prospective multicenter study in 33 private-sector Netcare hospitals within South 
Africa. AMS was implemented in 2012 at all sites that included noninfectious disease (ID) pharmacist-initiated review of antimicrobial therapy for basic interventions such as duration of therapy greater than 7 or 14 days, inappropriate duplicate antimicrobial coverage and if cultures were obtained prior to antimicrobial therapy. The review of hang-time compliance as an addition to Netcare's AMS prospective audit and feedback strategy was supported by the director of quality and received approval from each individual hospital's antibiotic stewardship committee prior to the study. The study received the necessary ethics approval retrospectively from the Netcare research committee as part of a quality improvement initiative.

A 4-week pilot study was conducted in November 2012 in two sites, both early adopters of AMS, and combined included 90 intensive care beds. These sites were chosen to undertake the pilot because of pharmacist time availability in wards and AMS capability. The pilot pharmacists established the methodology for data collection of the necessary metrics required to establish (1) the time of antibiotic prescription and (2) the time of antibiotic IV administration to calculate antibiotic hang-time. The time of antibiotic prescription was obtained from nursing notes that documented the time the physician assessed the patient and wrote the antibiotic order if this was not evident adjacent to the antibiotic prescription. As the study progressed, an antibiotic order form was implemented at 27 sites and included a designated place to document the time of the antibiotic prescription and the administration time. Pharmacists employed the Institute of Healthcare Improvement Principle of Plan, Do, Study, Act (PDSA) cycles to improve compliance [5]. Pharmacists conducted daily AMS rounds and evaluated the hang-time compliance of patients in the ICU and ICU step-down wards. At the start of 2014, each pharmacist had a goal to expand hang-time review to include general wards in each hospital. As each pharmacists' confidence and skill set improved, the opportunity arose to expand the program and ensure patients in step-down wards requiring antimicrobial therapy were also compliant with the hang-time measure and not only those in the ICUs.

Inclusion criteria included all patients' prescription charts in selected wards that commenced new antibiotic therapy (first dose of day 1). Pharmacists prospectively assessed the hang-time Monday-Friday during ward rounds within normal working hours. Those patients who commenced therapy after hours were assessed retrospectively the following business day. Exclusion criteria included single-dose antibiotic orders for surgical prophylaxis and patient charts where the time of antibiotic prescription was omitted that did not allow for the calculation of hang-time. Hang-time was defined as the time elapsed from the written antibiotic order to actual IV administration. 'Hang-time compliant' was defined as antibiotic administration within an elapsed time of $1 \mathrm{~h}$ from the written antibiotic order. To calculate the change in hang-time compliance pre-intervention was defined as week 1 and post-intervention as week 60. The Netcare AMS study coordinator was responsible for the implementation, education and real-time coordination of data with feedback to all 33 sites. Pharmacists collected hang-time data daily and reported it to the study coordinator in weekly intervals on a standardized data collection template using Microsoft Excel ${ }^{\circledR}$. A run chart reflecting weekly compliance with hang-time was generated. Study results from all sites were shared monthly by the study coordinator to create collaboration for excellence among sites. 
Hang-time results were presented monthly to each participating ward by pharmacists and discussed at hospital AMS committee meetings as a reminder of compliance and continuous monitoring. Instances of nonadherence to hang-time were addressed immediately with the nurse caring for the patient and escalated to the ward manager if necessary. The reasons as to why this project was important were constantly reinforced when noncompliance was noted by pharmacists.

Following the pilot study, hang-time compliance assessment was initiated in 33 hospitals using the tools of healthcare improvement spread methodology. This multisite implementation consisted of five stages, and an implementation toolkit was developed (Fig. 1). Stage 0 established the starting date, buy-in and support, and a "how to" guide. Stage 1 required all hospitals to complete a baseline hang-time compliance rate. Stage 2 provided educational in-services by pharmacists to the nursing staff and doctors. Educational posters were hung in nursing units to increase awareness of hang-time. Stage 3 consisted of daily data collection by the pharmacists. Stage 4 was submission of data monthly to the study coordinator who provided on-going feedback and support to all 33 sites. The study time frame was from 1 July 2013-30 August 2014.

Outcome data included the hang-time compliance pre-intervention at baseline (week $1)$, which was compared to compliance at the end of the study post-intervention (week 60). Compliance was calculated as the number of

\begin{tabular}{l|l}
\hline Stage $\mathbf{0}$ & - A guide detailing 'how to' implement the phases, data collection criteria and the context of the \\
study was written and distributed to all pharmacists participating at each hospital. \\
- Pharmacy Manager buy-in and support was obtained \\
- A hang-time educational poster was designed \\
- A start date was set for the study to commence across 33 hospitals
\end{tabular}

Fig. 1 The antibiotic 'hang-time' implementation tool kit for 33 hospitals 
patients who received day 1 antibiotics within $1 \mathrm{~h}$ divided by the total number of patients reviewed who were prescribed day 1 antibiotics. Compliance was expressed as a percentage. Process barriers resulting in a delay of antimicrobial administration within $1 \mathrm{~h}$ were identified and addressed separately by the responsible role players as a mechanism to enhance compliance. Pre- and post-intervention mortality rates were compared for all ICU patients, ICU patients on antibiotics, overall hospital mortality and all hospital patients who received antibiotics.

Statistical analysis was done using a two-tailed Fisher's exact test and chi-squared test with Yates correction where appropriate.

\section{RESULTS}

A total of 32,985 patients who received day 1 IV antibiotics were assessed for hang-time compliance from 33 hospitals over a 60-week study period. During this period, 21,069 patients received antibiotics within an hour following prescription and were assessed as hang-time compliant. The change in improvement of hang-time compliance of IV antimicrobial administration within $1 \mathrm{~h}$ following implementation of an AMS pharmacist-driven hang-time process improvement protocol was $41.2 \%$ pre-intervention $\quad(164 / 398)$ to $78.4 \%$ post-intervention $\quad(480 / 612 ; \quad P<0.0001)$. Table 1 provides data according to hospital size.

Figure 2 indicates the weekly percentage of patients compliant to hang-time for all 33 hospitals over the study period. Pharmacists reviewed twice as many patients during the final 4 weeks of the study (weeks 57-60; $n=1680$ ) compared to the first 4 weeks (weeks $1-4 ; n=834 ; P<0.0001)$. The majority (70\%) of the 33 hospitals achieved their goal of increasing the number of patients reviewed for hang-time compliance to include additional general wards in each hospital.

The following process barriers that contributed to hang-time noncompliance were addressed through education of nursing staff and consistent daily interventions by the pharmacists. Initially, it was difficult to obtain nursing buy-in to administer a new first dose antibiotic within an hour of the order because of routine fixed medicine dosing schedules, e.g., 2 a.m., 6 a.m., 10 a.m., 2 p.m., 6 p.m. and 10 p.m. If prescribers did not write "stat" on the first dose of antibiotic prescription, then the nurse thought the antibiotic was not urgent and would default the antibiotic administration to the fixed dosing schedule. In addition, if "stat" doses were not requested by the nurse, the

Table 1 Hospital demographics and number of patients reviewed by pharmacists for hang-time compliance within $1 \mathrm{~h}$

\begin{tabular}{lllcc}
\hline Hospital size & $\begin{array}{l}\text { Number of } \\
\text { hospital beds }\end{array}$ & $\begin{array}{l}\text { Netcare } \\
\text { hospitals }\end{array}$ & $\begin{array}{l}\text { Total number } \\
\text { of ICU beds }\end{array}$ & $\begin{array}{l}\text { Total number of patients } \\
\text { reviewed for 'hang-time' }\end{array}$ \\
\hline Large & $>300$ & 6 & 296 & 7643 \\
Medium/large & $200-299$ & 14 & 439 & 12,746 \\
Medium & $100-199$ & 11 & 164 & 9998 \\
Small & $<100$ & 2 & 11 & 2598 \\
Total & - & 33 & 910 & 32,985 \\
\hline
\end{tabular}

$I C U$ Intensive care unit 


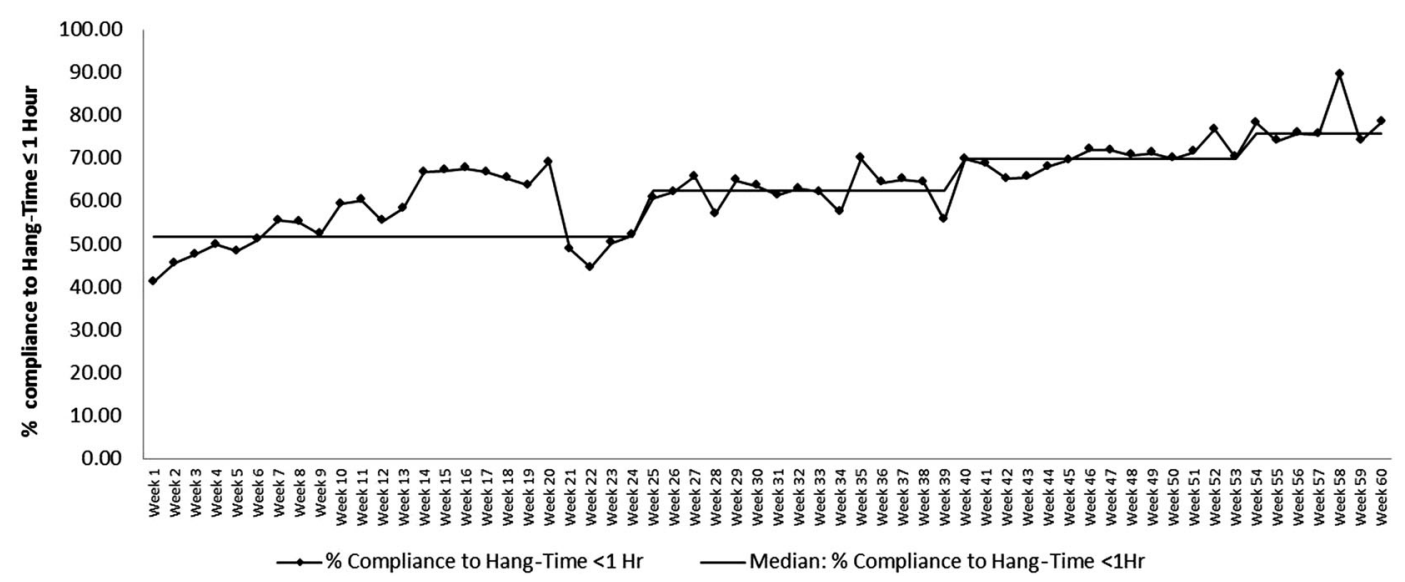

Fig. 2 Weekly antimicrobial hang-time compliance from 32,985 patients reviewed

pharmacy did not fast track the dispensing process. In an effort to improve delays with hang-time, pharmacy managers conducted a utilization review of the most commonly prescribed antimicrobials per ward and allocated stock of these agents to be kept as part of the units' medication ward stock. This was instituted in order to circumvent delays in the dispensing process for the initial dose. The process in the pharmacy for 'stat' doses was also revised and a 'stat' basket or window was created in some pharmacies to ensure these prescriptions were dispensed as a priority.

Mortality data were analyzed comparing mortality in July 2013 to August 2014. No statistical significance was found when analyzing the overall ICU mortality rate $(P=0.2702)$, patients on antibiotics in the ICU $(P=0.186), \quad$ overall hospital mortality $(P=0.6624)$ and all patients who received antibiotics $(P=0.7598)$.

\section{DISCUSSION}

To our knowledge this is the first study to demonstrate a successful national AMS process improvement initiative led by non-ID pharmacists in a large network of hospitals in a resource-limited country. A sustained improvement in hang-time compliance during the 60-week study period was observed. The measurement of hang-time was included as a fundamental measure in basic stewardship review and has been mandated as a standard performance measure of pharmacists performing AMS assessment. Therefore, compliance with hang-time continues to be reported to the project manager and to date (July 2015) continues to be successfully sustained. Our findings suggest that within healthcare systems with limited resources, pharmacists are key role players within a multidisciplinary team and are able to drive AMS programs and impact patient care. Most published data on AMS in hospital settings come from experiences in academic centers and tend to represent single-center studies. In contrast, our study had 33 sites from community hospitals without ID-trained physicians or ID pharmacists. Ideally AMS programs should be directed by an ID physician and a clinical pharmacist with ID training; however, in South Africa this is not possible because of the lack of training programs for ID pharmacists and low numbers of ID physicians. 
There are only 13,579 registered pharmacists in South Africa who work in inpatient, outpatient and retail pharmacy [6]. The introduction of clinical pharmacy practice is in its infancy and creates an additional barrier for pharmacists and AMS. Although the barriers may not be as big, other countries describe similar resource challenges. Trevidi et al. compared hospital AMS infrastructure in the UK, France and the US [7]. In France, there is no national curriculum to train ID pharmacists. As AMS expands, ID expertise is not available in all UK or US community non-academic facilities. We believe our successful outcome of improved hang-time compliance was largely due to stage 4 of the project implementation toolkit, including the importance of individual hospital feedback, hospital ranking in relation to others, repetition of pharmacist initiated education and training to nursing staff and prescribers at the point of care, regular feedback of results to the patient care wards and the collaborative environment created by the pharmacist study coordinator between each of the hospitals and wards. The provision of support, quality improvement guidance and real-time feedback to pharmacists by the study coordinator was important to maintaining the momentum and rigor of the intervention. The launch of this hang-time initiative also created the desired awareness and collaboration among doctors, nurses, pharmacists and management that is necessary to initiate additional AMS initiatives in our hospital network.

Numerous publications support the prompt administration of antimicrobials to enhance patient quality outcomes [1-3, 8-11]. Kumar et al. published the first study that showed the relationship between mortality and timing of administration of antibiotics in patients with new-onset hypotension and septic shock. Each hour of delay in antimicrobial administration was associated with an average increase in mortality of $7.6 \%$. Only $14.5 \%$ of patients studied received antibiotics within $1 \mathrm{~h}$ of the documented onset of hypotension, and the median time to administer antimicrobial therapy was $6 \mathrm{~h}$ [1]. Our study showed that $78.4 \%$ of patients reviewed received their antibiotic within $1 \mathrm{~h}$ following written prescriptions and pharmacist intervention. In a large multicenter study of 28,150 patients with severe sepsis and septic shock, Ferrer and colleagues also found that hourly delays in antibiotic administration have a significant impact on mortality across all areas in the hospital, not just in ICU patients. The focus was on time delays and the association with mortality and not on the number of patients who received an antibiotic within $1 \mathrm{~h}$ [2]. The patient populations in both the Kumar and Ferrer studies included patients with septic shock at the time of onset of hypotension and patients with severe sepsis and septic shock, respectively $[1,2]$. Due to limited documentation of patient diagnosis in the paper charts, our study did not consider the patient diagnosis and strived to ensure that all patients who were prescribed an antibiotic received it within an hour regardless of diagnosis, severity of illness and location in the hospital. This is in line with the recommendations made by Ferrer et al. of timely antibiotic administration leading to better outcomes across a varied spectrum of hospital patients [2]. Additional studies evaluating the timeliness of antimicrobials and impact on outcomes in patients with community-acquired pneumonia and orthopedic surgical procedures supports our initiative's decision not to limit the role of AMS pharmacists' involvement in hang-time interventions to only patients with sepsis or septic shock $[8,9]$. 
Although not an objective of the study, we were unable to show a significant impact on patient mortality. Reasons for this may include that the appropriateness of antibiotic therapy was not evaluated and all patients reviewed were included in the study, not just those with sepsis and septic shock. Similarly, Ferrer et al. did not assess antibiotic appropriateness although Kumar and team focused on time to effective antibiotic $[1,2]$. Studies also exist that have not established a correlation between the time of the first dose antimicrobial to positive clinical outcomes and decreased mortality. This is evidenced by both Bordon et al. and Chung and Buising when evaluating patients with community-acquired pneumonia, which may suggest that the correct diagnosis and selection of the appropriate agent may have an enhanced influence on patient mortality rather than time of administration $[8,12]$.

The increasing rates of MDR organisms and poor compliance with prescribing the appropriate empiric antibiotic in South African hospitals could be additional contributing factors to consider when noting the absence of a difference in patient mortality in our study [13]. This study has highlighted an important additional role for pharmacists in driving AMS programs in hospitals. We believe good AMS including the prompt administration of appropriate antimicrobials and pharmacist intervention at the point of care can have a valuable influence on patient care. Additional AMS indications that could impact patient outcomes include initiatives such as inappropriate extended antimicrobial duration, concurrent duplicate antimicrobial cover, cultures not done prior to the initiation of empiric therapy and the administration of more than four antibiotics simultaneously. A study is currently being conducted to evaluate the non-ID trained pharmacists' role in these low-hanging-fruit initiatives within our hospital network.

Our study has limitations. First the main goal of the study was to evaluate hang-time compliance with initial antibiotic administration and not appropriateness. Inappropriate, unnecessary or inadequate antibiotics may confound our results. Second, the hospitals use paper charts and results may not be applicable to hospitals using electronic physician order entry. Third, the hospital network is a private sector network with strong line management systems in place and a director who oversees AMS initiatives across all hospitals. Gaieski and colleagues recommend placing high priority on developing systems to ensure the prompt administration of antimicrobials [11]. We believe that our study was able to achieve this successfully in multiple hospital sites without electronic dispensing systems. This methodology could be applied to other hospital networks in limited-resource countries as a model to implement this AMS hang-time initiative.

\section{CONCLUSIONS}

Timely administration of antimicrobials should be considered a low-hanging-fruit component of AMS. Non-ID pharmacists can lead interdisciplinary engagement between doctors and nurses to strengthen the importance of early administration of antimicrobials to improve patient care. The role of the non-ID pharmacist in driving an AMS program across a health system network cannot be underestimated, and the importance of coordination as well as real-time feedback of data to the front line staff is a key strategy to obtain sustained buy-in. 


\section{ACKNOWLEDGMENTS}

This supplement was not sponsored by outside commercial interests. Partial support for this work was provided by The Ohio State University Outreach and Engagement Grant. No source of funding was received for publication of this article. All named authors meet the International Committee of Medical Journal Editors (ICMJE) criteria for authorship for this manuscript, take responsibility for the integrity of the work as a whole and have given final approval to the version published. We wish to thank all Netcare pharmacists who participated in this work, their pharmacy managers for their support and other members of the healthcare teams including doctors and nursing staff for their efforts and contributions to making this study achievable.

Conflict of interest. Angeliki Messina and Dena van den Bergh are employees of Netcare Hospitals, Ltd., and have no conflicts of interest. Debra Goff has nothing to disclose.

Compliance with ethics guidelines. Ethics clearance for this study was obtained retrospectively from Pharma-Ethics and the Netcare institutional review committee. This study conformed with the Helsinki Declaration of 1964, as revised in 2013.

Open Access. This article is distributed under the terms of the Creative Commons Attribution-NonCommercial 4.0 International License (http://creativecommons.org/licenses/ by-nc/4.0/), which permits any noncommercial use, distribution, and reproduction in any medium, provided you give appropriate credit to the original author(s) and the source, provide a link to the Creative Commons license, and indicate if changes were made.

\section{REFERENCES}

1. Kumar A, Roberts D, Wood KE, et al. Duration of hypotension before initiation of effective antimicrobial therapy is the critical determinant of survival in human septic shock. Crit Care Med. 2006;34:1589-96.

2. Ferrer R, Martin-Loeches I, Phillips G, et al. Empiric Antibiotic treatment reduces mortality in severe sepsis and septic shock from the first hour: results from a guideline-based performance improvement program. Crit Care Med. 2014;42(8):1749-55.

3. Cohen J, Vincent JL, Adhikari NKJ, et al. Sepsis: a roadmap for future research. Lancet Infect Dis. 2015;15:581-614.

4. Dellit TH, Owens RC, McGowan JE Jr, et al. Infectious Diseases Society of America and the Society for Healthcare Epidemiology of America guidelines for developing an institutional program to enhance antimicrobial stewardship. Clin Infect Dis. 2007;44:159-77.

5. Varkey P, Reller K, Resar RK. Basics of quality improvement in healthcare. Mayo Clinic Proc. 2007;82(6):735-9.

6. The South African Pharmacy Council. 2015. Available at http://www.pharmcouncil.co.za/B_ StatsPerByGender.asp. Accessed Mar 27, 2015.

7. Trivedi KK, Dumartin C, Gilcrest $M$, Wade $P$, Howard P. Identifying best practices across three countries: hospital antimicrobial stewardship in the United Kingdom, France, and the United States. Clin Infect Dis. 2014;59(S3):S170-8.

8. Bardon J, Aliberti S, Duvvuri P, et al. Early administration of the first antimicrobials should be considered a marker of optimal care of patients with community-acquired pneumonia rather than a predictor of outcomes. Int $\mathrm{J}$ Infect Dis. 2013;17:293-8.

9. Lack WD, Karunakar MA, Angerame MR, et al. Type III open Tibia fractures: immediate antibiotic prophylaxis minimizes infection. J Orthop Trauma. 2015;29(1):1-6.

10. Van Zanten ARH. The golden hour of antibiotic administration in severe sepsis: avoid a false start striving for gold. Crit Care Med. 2014;42(8):1931-2.

11. Gaieski DF, Pines JM, Band RA, et al. Impact of time to antibiotics on survival in patients with severe sepsis or septic shock in whom early goal-directed therapy was initiated in the emergency department. Crit Care Med. 2010;38(3):1-9. 
12. Chung AC, Buising KL. Delayed administration of antibiotics and mortality in patients with community-acquired pneumonia. Ann Emerg Med. 2009;53(5):6128-624.

13. Paruk F, Richards G, Scribante J, Bhagwanjee S, Mer $\mathrm{M}$, Perrie H. Antibiotic prescription practices and their relationship to outcome in South African intensive care units: findings of the prevalence of infection in South African intensive care units (PISA) study. S Afr Med J. 2012;102(7):613-6. 